Recibido: 25/05/2020 --- Aceptado: 12/09/2020 --- Publicado: 12/04/2021

\title{
SCHOOLOGY: PLATAFORMA WEB CAPAZ DE MEJORAR EL PROCESO DE ENSEÑANZA-APRENDIZAJE EN EL NIVEL EDUCATIVO SUPERIOR
}

\section{SCHOOLOGY: A WEB PLATFORM CAPABLE OF IMPROVING THE TEACHING-LEARNING PROCESS AT THE HIGHER EDUCATIONAL LEVEL}

(D) Ricardo-Adán Salas-Rueda. ${ }^{\mathbf{1}}$ Universidad Nacional Autónoma de México. México.

ricardo.salas@icat.unam.mx

Carlos-Alberto Jiménez-Bandala: Universidad La Salle. México. carlos jimenez@lasalle.mx

Clara Alvarado-Zamorano: Universidad Nacional Autónoma de México. México. clara.alvarado@icat.unam.mx

\section{Cómo citar el artículo:}

Salas-Rueda, R. A., Jiménez-Bandala, C-A. y Alvarado-Zamorano, C. (2021). Schoology: plataforma web capaz de mejorar el proceso de enseñanza-aprendizaje en el nivel educativo superior. Revista de Comunicación de la SEECI, 54, 19-41. http://doi.org/10.15198/seeci.2021.54.e645

\section{RESUMEN}

El objetivo de esta investigación cuantitativa y cualitativa es analizar el impacto de Schoology en la asignatura Taller de Investigación, durante la creación de un artículo científico considerando la ciencia de datos. Schoology es una plataforma web gratuita que permite la creación y difusión de los contenidos de los cursos, el diseño de las lecciones, la comunicación entre los participantes y la evaluación de las actividades escolares. La muestra está compuesta por 27 estudiantes que cursaron las Licenciaturas en Administración, Comercio, Contaduría y Mercadotecnia en una universidad privada de la Ciudad de México durante el ciclo escolar 2017. Los resultados del aprendizaje automático (regresión lineal) indican que la facilidad de uso y la interacción en esta plataforma influyen positivamente la comunicación, la

\footnotetext{
${ }^{1}$ Ricardo-Adán Salas-Rueda: Doctor en Diseño de Nuevas Tecnologías. Investigador de Tiempo Completo en el Instituto de Ciencias Aplicadas y Tecnología, Universidad Nacional Autónoma de México. Investigador Nacional SNI nivel 1 (CONACYT).
} 
Salas-Rueda, R. A., Jiménez-Bandala, C-A. y Alvarado-Zamorano, C.

Schoology: plataforma web capaz de mejorar el proceso de enseñanza-aprendizaje en el nivel educativo superior

colaboración y el rol activo de los estudiantes, durante la creación del artículo científico. La ciencia de datos identifica 6 modelos predictivos sobre el uso de Schoology en el campo educativo. El enfoque cualitativo indica que sus foros de discusión tienen un papel fundamental durante el proceso de enseñanza-aprendizaje. Además, las ventajas de Schoology son la entrega de las tareas en la plataforma, realización de evaluaciones online y consulta de la información de los cursos en cualquier momento y lugar. Por último, los docentes tienen la oportunidad de construir nuevos espacios virtuales educativos por medio de Schoology.

PALABRAS CLAVE: Tecnología - enseñanza superior - TIC - aprendizaje - ciencia de datos - aprendizaje máquina - educación a distancia

\section{ABSTRACT}

The objective of this quantitative and qualitative research is to analyze the impact of Schoology in the Research Workshop course, during the creation of a scientific article considering data science. Schoology is a free web platform that allows the creation and dissemination of the course contents, lesson design, communication between the participants and evaluation of the school activities. The sample is composed of 27 students who took the Bachelor of Administration, Commerce, Accounting and Marketing at a private university in Mexico City during the 2017 school year. The results of machine learning (linear regression) indicate that the ease of use and interaction in this platform positively influence the communication, collaboration and active role of the students, during the creation of the scientific article. Data science identifies 6 predictive models about the use of Schoology in the educational field. The qualitative approach indicates that their discussion forums have a fundamental role during the teaching-learning process. In addition, the advantages of Schoology are the delivery of tasks on the platform, conducting online evaluations and consulting the information of the courses at any time and place. Finally, teachers have the opportunity to build new educational virtual spaces through Schoology.

KEY WORDS: Technology - higher level education - ICT - learning - data science machine learning - distance education.

\section{SCHOOLOGY: PLATAFORMA WEB CAPAZ DE MELHORAR O PROCESSO DE ENSINO-APRENDIZAGEM NO NÍVEL SUPERIOR EDUCACIONAL.}

\section{RESUMO}

O objetivo desta pesquisa quantitativa e qualitativa é analisar o impacto de Schoology na disciplina de Taller de Investigación, durante a criação de um artigo científico considerando a ciência de dados. Schoology é uma plataforma web gratuita que permite a criação e difusão dos conteúdos dos cursos, a estrutura das aulas, a comunicação entre os participantes e a avaliação das atividades escolares. A mostra é composta por 27 estudantes que fizeram as Licenciaturas em Administração, 
Salas-Rueda, R. A., Jiménez-Bandala, C-A. y Alvarado-Zamorano, C.

Schoology: plataforma web capaz de mejorar el proceso de enseñanza-aprendizaje en el nivel educativo superior

Comércio, Contabilidade e Marketing em uma universidade privada da Cidade de México no ciclo letivo de 2017. Os resultados do aprendizado automático (regressão linear) indicam que a facilidade de uso e a interação nesta plataforma influenciam positivamente a comunicação, a colaboração e o papel ativo dos estudantes, durante a criação do artigo científico. A ciência de dados identifica 6 modelos preditivos sobre o uso de Schoology no campo educativo. $O$ foco qualitativo indica que seus fóruns de discussão têm um papel fundamental durante o processo de ensino-aprendizagem. Além disso, as vantagens de Schoology são a entrega dos deveres de casa na própria plataforma, realização de avaliações online e consulta da informação dos cursos em qualquer momento e lugar. Por último, os professores têm a oportunidade de construir novos espaços virtuais educativos por meio de Schoology.

PALABRAS CHAVE: Tecnología - ensino superior - TIC - aprendizado - ciencia de dados - aprendizado de máquina - educação a distância

\section{INTRODUCCIÓN}

Hoy en día, las instituciones educativas necesitan desarrollar nuevos planes de estudio, estrategias didácticas y modelos pedagógicos que faciliten el proceso de enseñanza-aprendizaje por medio de la tecnología (Aldalalah, Ababneh, Bawaneh y Alzubi, 2019; Deng y Tavares, 2013; Salas-Rueda, 2019). Las Tecnologías de la Información y Comunicación (TICs) facilitan la realización de las actividades escolares y tareas, incrementan la participación entre los estudiantes y mejoran la compresión de los temas de los cursos (Yundayani, Kardijan y Herawan, 2019). Por ejemplo, Tazouti, Boulaknadel y Fakhri (2019) explican que el uso de las aplicaciones y plataformas web en el campo educativo permite el desarrollo de las competencias en los estudiantes.

Para lograr el uso eficiente de la tecnología dentro y fuera del salón de clases, los docentes necesitan identificar y establecer los objetivos del curso y planear las actividades instruccionales (Dewi, Lengkanawati y Purnawarman, 2019). En particular, el modelo Technology, Pedagogy And Content Knowledge (TPACK) permite la integración de las herramientas digitales en las actividades escolares y el uso de las plataformas web educativas por medio de los conocimientos disciplinares, tecnológicos y pedagógicos (Dewi, Lengkanawati y Purnawarman, 2019).

El rápido desarrollo de las TICs está provocando la planeación y construcción de nuevos escenarios para el aprendizaje y la enseñanza (Bhagat, Wu y Chang, 2019; Zhang y Li, 2019). En particular, las plataformas web permiten innovar el proceso de enseñanza-aprendizaje por medio de la consulta de la información en cualquier momento y lugar, la comunicación de los participantes, la entrega de actividades escolares y el uso de las herramientas tecnológicas (Boloudakis, Retalis y Psaromiligkos, 2018).

En el Siglo XXI, los docentes están incorporando diversas plataformas web como Moodle (Chung y Ackerman, 2015; Hendra-Divayana, 2019; Zou, Liu y Yang, 2012), Blackboard (Elmaadaway, 2017), Edmodo (Cao y Liu, 2019; Hendra-Divayana, 2019) 
Salas-Rueda, R. A., Jiménez-Bandala, C-A. y Alvarado-Zamorano, C.

Schoology: plataforma web capaz de mejorar el proceso de enseñanza-aprendizaje en el nivel educativo superior

y Kelase (Hendra-Divayana, 2019) en las actividades escolares, con el propósito de mejorar e innovar el proceso de enseñanza-aprendizaje y desarrollar las competencias de los estudiantes.

Schoology es una plataforma web que permite la creación y difusión de los contenidos de los cursos, el diseño de las lecciones, la comunicación entre los participantes y la evaluación de las actividades escolares. Por lo tanto, esta investigación mixta tiene como objetivo analizar el impacto de Schoology en la asignatura Taller de Investigación, durante la creación de un artículo científico considerando la ciencia de datos. Las preguntas de investigación fueron:

- ¿Cuál es el impacto de Schoology (facilidad de uso e interacción) en el proceso de enseñanza-aprendizaje?

- ¿Cuáles son los modelos predictivos sobre el uso de Schoology durante el proceso educativo (comunicación, colaboración y rol activo de los estudiantes)?

- ¿Cuáles son las percepciones de los alumnos sobre el uso de Schoology en la asignatura Taller de Investigación?

\subsection{Uso de las TICs en el campo educativo}

Las estrategias de enseñanza-aprendizaje están sufriendo modificaciones en todos los niveles educativos a causa de la incorporación de la tecnología, dentro y fuera del salón de clases (Hendra-Divayana, 2019; Parsons, Inkila y Lynch, 2019). Por ejemplo, el uso de dispositivos móviles se está incrementando en los cursos presenciales y a distancia debido a que los alumnos pueden acceder a las aplicaciones y plataformas web en cualquier momento y lugar (Byrne, 2019).

Incluso, los celulares inteligentes y laptops facilitan la implementación del aula invertida en el contexto educativo (Byrne, 2019). En particular, Elmaadaway (2017) organizó y realizó diversas actividades antes, durante y después de la sesión presencial (aula invertida) en la plataforma web Blackboard, con el propósito de facilitar el proceso de enseñanza-aprendizaje sobre la electrónica.

El empleo de las herramientas tecnológicas en el campo educativo mejora la calidad y permite la creación de espacios innovadores para la enseñanza y el aprendizaje (Dewi, Lengkanawati y Purnawarman, 2019). En particular, Aldalalah, Ababneh et al. (2019) proponen el uso de la tecnología (p.ej., realidad aumentada, plataformas web y simuladores) para mejorar el rendimiento académico y pensamiento visual de los estudiantes durante los cursos de las matemáticas.

De hecho, los avances de la tecnología permiten crear nuevos espacios para el aprendizaje y la enseñanza (Salas-Rueda, 2018). En particular, las plataformas web permiten mejorar el proceso educativo a través de la consulta de contenidos, realización de los cuestionarios en línea y participación en los foros de discusión ( $\mathrm{Li}$, Ouyang y Yang, 2019). 
Salas-Rueda, R. A., Jiménez-Bandala, C-A. y Alvarado-Zamorano, C.

Schoology: plataforma web capaz de mejorar el proceso de enseñanza-aprendizaje en el nivel educativo superior

\subsection{Uso de las plataformas web educativas}

Hoy en día, las plataformas web como Moodle y Edmodo facilitan la planeación, organización e implementación de las actividades en los cursos bajo la modalidad Blended learning (Hendra-Divayana, 2019). Blended learning es una modalidad que promueve la realización de diversas actividades a distancia y en el salón de clases por medio del uso de las plataformas web (Hendra-Divayana, 2019).

La incorporación de la plataforma web Edmodo en los cursos de Inglés incrementa la motivación, mejora el rendimiento académico y facilita la comunicación de los estudiantes (Cao y Liu, 2019). Asimismo, Hendra-Divayana (2019) propone el empleo de la plataforma Kelase en la modalidad Blended learning para compartir los contenidos de los cursos, realizar las actividades colaborativas, mejorar la comunicación entre los estudiantes y facilitar la participación en los foros de discusión.

Por otro lado, el uso de Moodle como herramienta de apoyo durante el proceso de enseñanza-aprendizaje mejora el rendimiento académico de los estudiantes en el nivel educativo básico (Zou, Liu y Yang, 2012). Incluso, Moodle influye positivamente la asimilación del conocimiento y facilita el trabajo colaborativo (Dias, Hadjileontiadou, Diniz y Hadjileontiadis, 2017).

En la actualidad, las universidades están incrementando el uso de las plataformas web (p.ej., Moodle), con el propósito de desarrollar las competencias de los estudiantes (Cerezo, Sánchez-Santillán, Paule-Ruiz y Núñez, 2016). De hecho, los docentes pueden construir nuevos ambientes virtuales educativos de forma sencilla y eficiente por medio de las plataformas web (Costello, 2013; Elmaadaway, 2017; Yalcin y Kutlu, 2019). Por ejemplo, Moodle facilita la interacción y comunicación entre los alumnos y el docente (Chung y Ackerman, 2015). Incluso, esta plataforma web permite el desarrollo de las habilidades en los estudiantes (Boloudakis, Retalis y Psaromiligkos, 2018).

En el Siglo XXI, las plataformas web están transformado la planeación de los cursos por medio de la realización de diversas actividades escolares dentro y fuera del salón de clases (Hendra-Divayana, 2019; Li, Ouyang y Yang, 2019). Incluso, el uso de las plataformas web en el campo educativo favorece el desarrollo de las competencias en los estudiantes y mejora el proceso de enseñanza-aprendizaje (Boloudakis, Retalis y Psaromiligkos, 2018; Cerezo et al., 2016; Dias et al., 2017).

\section{OBJETIVOS}

Los objetivos particulares de la investigación son (1) analizar el impacto de Schoology (facilidad de uso e interacción) en el proceso de enseñanza-aprendizaje (2) identificar los modelos predictivos sobre el uso de Schoology durante el proceso educativo (comunicación, colaboración y rol activo de los estudiantes) y (3) analizar las percepciones de los alumnos sobre el uso de Schoology en la asignatura Taller de Investigación. 


\section{METODOLOGÍA}

Esta investigación mixta tiene como objetivo analizar el impacto de Schoology en la asignatura Taller de investigación durante la creación de un artículo científico considerando la ciencia de datos. El enfoque cuantitativo permite analizar el impacto de Schoology en el proceso de enseñanza-aprendizaje y el enfoque cualitativo permite analizar las percepciones de los alumnos sobre el uso de Schoology en la asignatura Taller de Investigación. Las ventajas sobre el empleo de Schoology en el campo educativo son la facilidad de uso, sencillez de su interfaz y disponibilidad de la información en cualquier momento y lugar.

\subsection{Participantes}

La muestra está compuesta por 27 estudiantes que cursaron las Licenciaturas en Administración, Comercio, Contaduría y Mercadotecnia en una universidad privada de la Ciudad de México, durante el ciclo escolar 2017.

\subsection{Procedimiento}

Durante 10 semanas, los estudiantes de las Licenciaturas en Administración, Comercio, Contaduría y Mercadotecnia utilizaron Schoology, con el propósito de facilitar la elaboración de un artículo científico.

Schoology es una plataforma web que permite la creación y difusión de los contenidos de los cursos, el diseño de las lecciones, la comunicación entre los participantes y la evaluación de las actividades escolares. Esta aplicación tecnológica está disponible en la siguiente dirección web: https://www.schoology.com/

La Figura 1 muestra el modelo de aceptación tecnológica sobre el uso de Schoology en el campo educativo.

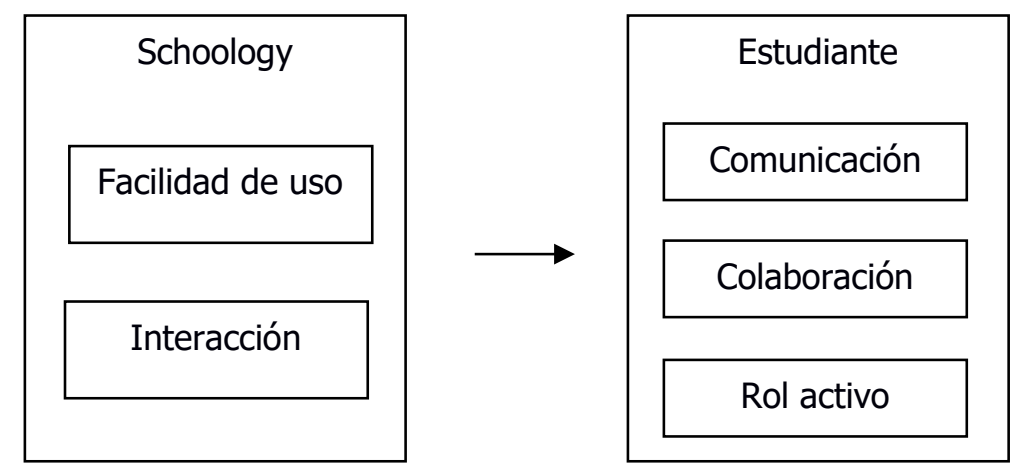

Figura 1. Modelo de aceptación sobre el uso de Schoology.

Fuente: Elaboración propia. 
Salas-Rueda, R. A., Jiménez-Bandala, C-A. y Alvarado-Zamorano, C.

Schoology: plataforma web capaz de mejorar el proceso de enseñanza-aprendizaje en el nivel educativo superior

La facilidad de uso se refiere a la eficacia en el manejo de las herramientas y aplicaciones tecnológicas y la interacción se refiere al intercambio de información entre los programas y las personas. Por otro lado, la comunicación se refiere a la realización de los foros de discusión y la consulta de los materiales, la colaboración se refiere a la elaboración de las actividades y el rol activo se refiere a la participación de los estudiantes durante el proceso educativo.

Las hipótesis sobre el uso de Schoology y la comunicación de los estudiantes son:

- Hipótesis 1 (H1): La facilidad de uso en Schoology influye positivamente la comunicación de los estudiantes durante la creación del artículo científico

- Hipótesis 2 (H2): La interacción en Schoology influye positivamente la comunicación de los estudiantes durante la creación del artículo científico

Las hipótesis sobre el uso de Schoology y la colaboración de los estudiantes son:

- Hipótesis 3 (H3): La facilidad de uso en Schoology influye positivamente la colaboración de los estudiantes durante la creación del artículo científico

- Hipótesis 4 (H4): La interacción en Schoology influye positivamente la colaboración de los estudiantes durante la creación del artículo científico

Las hipótesis sobre el uso de Schoology y el rol activo de los estudiantes son:

- Hipótesis 5 (H5): La facilidad de uso en Schoology influye positivamente el rol activo de los estudiantes durante la creación del artículo científico

- Hipótesis 6 (H6): La interacción en Schoology influye positivamente el rol activo de los estudiantes durante la creación del artículo científico

La ciencia de datos identificó los siguientes modelos predictivos por medio de la técnica árbol de decisión:

- Modelo predictivo 1 sobre la facilidad de uso en Schoology y la comunicación de los estudiantes durante la creación del artículo científico

- Modelo predictivo 2 sobre la interacción en Schoology y la comunicación de los estudiantes durante la creación del artículo científico

- Modelo predictivo 3 sobre la facilidad de uso en Schoology y la colaboración de los estudiantes durante la creación del artículo científico

- Modelo predictivo 4 sobre la interacción en Schoology y la colaboración de los estudiantes durante la creación del artículo científico

- Modelo predictivo 5 sobre la facilidad de uso en Schoology y el rol activo de los estudiantes durante la creación del artículo científico

- Modelo predictivo 6 sobre la interacción en Schoology y el rol activo de los estudiantes durante la creación del artículo científico

\subsection{Análisis de datos}

La herramienta Rapidminer permitió realizar el cálculo del aprendizaje automático (regresión lineal) para evaluar las hipótesis sobre el uso de Schoology en la asignatura Taller de Investigación. El aprendizaje automático permitió calcular la regresión lineal por medio de la sección entrenamiento $(60 \%, 70 \%$ y $80 \%$ de la 
muestra) e identificar la exactitud de la regresión lineal por medio de la sección evaluación ( $40 \%, 30 \%$ y $20 \%$ de la muestra).

Asimismo, la técnica árbol de decisión (ciencia de datos) permitió identificar los modelos predictivos sobre el uso de Schoology en la campo educativo, por medio de la herramienta Rapidminer (Ver Figura 2).

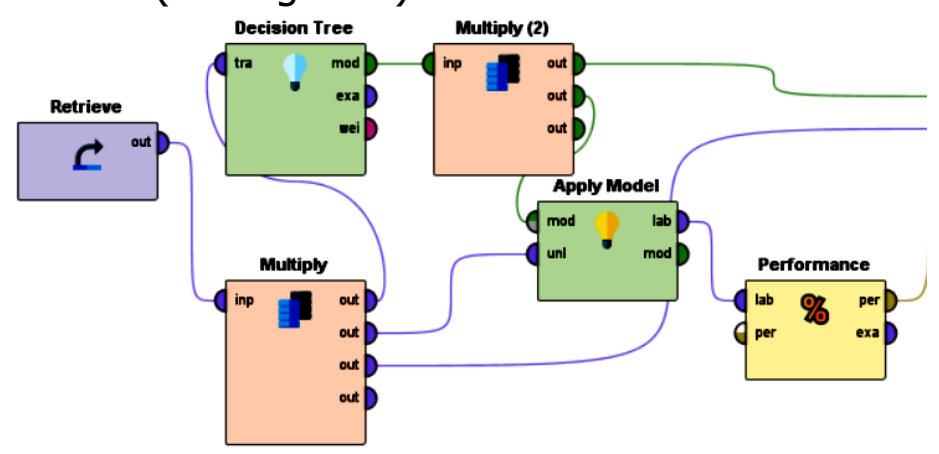

Figura 2. Uso de la herramienta Rapidminer.

Fuente: Elaboración propia por medio de la herramienta Rapidminer.

Además, el software Maxqda permitió la construcción de la nube de palabras sobre el uso de Schoology en el proceso de enseñanza-aprendizaje.

\subsection{Recolección de datos}

La Tabla 1 muestra el instrumento de medición (cuestionario) utilizado para recolectar la información sobre el impacto de Schoology en la asignatura Taller de investigación.

Tabla 1. Cuestionario.

\begin{tabular}{|c|c|c|c|c|c|c|}
\hline No. & Variable & Dimensión & Pregunta & Respuesta & $\mathbf{n}$ & $\%$ \\
\hline \multirow{14}{*}{1} & \multirow{14}{*}{$\begin{array}{l}\text { Perfil del } \\
\text { alumno }\end{array}$} & \multirow{3}{*}{ Sexo } & \multirow[t]{3}{*}{ 1.- ¿Cuál es tu sexo? } & & & \\
\hline & & & & Hombre & 16 & $59.26 \%$ \\
\hline & & & & Mujer & 11 & $40.74 \%$ \\
\hline & & \multirow{5}{*}{ Carrera } & \multirow[t]{5}{*}{ 2.- ¿Cuál es tu carrera? } & & & \\
\hline & & & & Administración & 5 & $18.52 \%$ \\
\hline & & & & Comercio & 8 & $29.63 \%$ \\
\hline & & & & Contaduría & 8 & $29.63 \%$ \\
\hline & & & & Mercadotecnia & 6 & $22.22 \%$ \\
\hline & & \multirow{6}{*}{ Edad } & \multirow[t]{6}{*}{ 3.- ¿Cuál es tu edad? } & & & \\
\hline & & & & 17 años & 1 & $3.70 \%$ \\
\hline & & & & 18 años & 13 & $48.15 \%$ \\
\hline & & & & 19 años & 8 & $29.63 \%$ \\
\hline & & & & 20 años & 2 & $7.41 \%$ \\
\hline & & & & 21 años & 3 & $11.11 \%$ \\
\hline \multirow{4}{*}{2} & \multirow{4}{*}{ Tecnología } & \multirow{4}{*}{ Schoology } & \multirow{4}{*}{$\begin{array}{l}\text { 4.- La facilidad de uso en } \\
\text { Schoology influye durante el } \\
\text { aprendizaje }\end{array}$} & & & \\
\hline & & & & Bastante (1) & 12 & $44.44 \%$ \\
\hline & & & & Mucho (2) & 10 & $37.04 \%$ \\
\hline & & & & Poco (3) & 4 & $14.81 \%$ \\
\hline
\end{tabular}


Salas-Rueda, R. A., Jiménez-Bandala, C-A. y Alvarado-Zamorano, C.

Schoology: plataforma web capaz de mejorar el proceso de enseñanza-aprendizaje en el nivel educativo superior

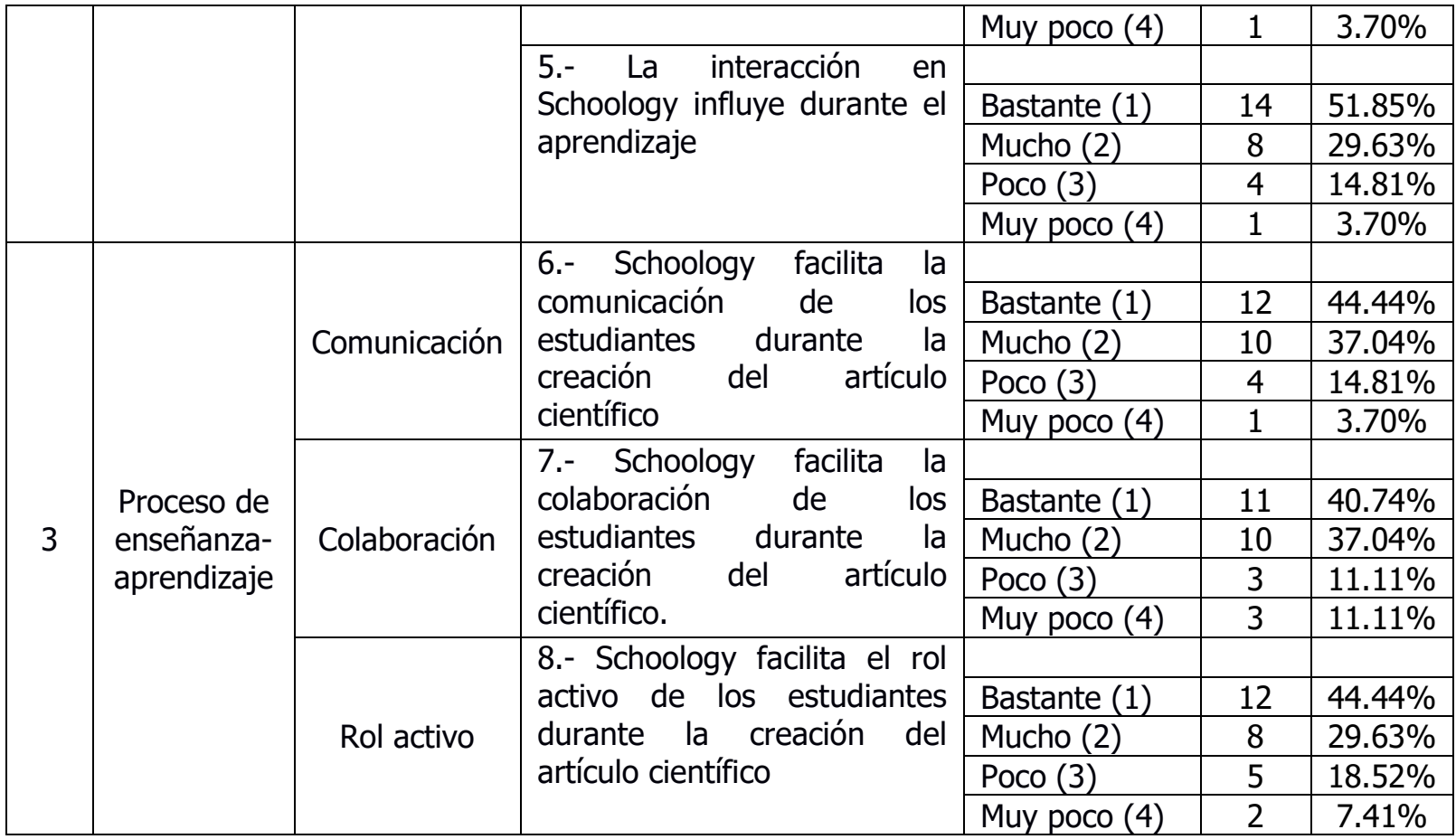

Fuente: Elaboración propia.

Además, el instrumento de medición consta de 4 preguntas abiertas sobre el impacto, las ventajas, la utilidad y el uso de Schoology en el campo educativo.

\section{RESULTADOS}

A continuación, se presentan los resultados obtenidos sobre el uso de Schoology en el proceso educativo de la asignatura Taller de Investigación, considerando la ciencia de datos. La Tabla 2 muestra los resultados del aprendizaje automático (regresión lineal) con $60 \%$, $70 \%$ y $80 \%$ de entrenamiento.

Tabla 2. Resultados de la regresión lineal.

\begin{tabular}{|c|c|c|c|c|c|}
\hline No. & Hipótesis & Entrenamiento & Evaluación & Función lineal & Conclusión \\
\hline \multirow{3}{*}{1} & \multirow{3}{*}{$\begin{array}{l}\text { H1: Facilidad de } \\
\text { uso en Schoology } \\
\rightarrow \text { comunicación }\end{array}$} & $60 \%$ & $40 \%$ & $y=0.326 x+1.240$ & Acepta: 0.326 \\
\hline & & $70 \%$ & $30 \%$ & $y=0.302 x+1.354$ & Acepta: 0.302 \\
\hline & & $80 \%$ & $20 \%$ & $y=0.257 x+1.394$ & Acepta: 0.257 \\
\hline \multirow{3}{*}{2} & \multirow{3}{*}{$\begin{array}{l}\text { H2: Interacción en } \\
\text { Schoology } \\
\text { comunicación }\end{array}$} & $60 \%$ & $40 \%$ & $y=0.326 x+1.240$ & Acepta: 0.326 \\
\hline & & $70 \%$ & $30 \%$ & $y=0.368 x+1.236$ & Acepta: 0.368 \\
\hline & & $80 \%$ & $20 \%$ & $y=0.336 x+1.235$ & Acepta: 0.336 \\
\hline \multirow{3}{*}{3} & \multirow{3}{*}{$\begin{array}{l}\text { H3: Facilidad de } \\
\text { uso en Schoology } \\
\rightarrow \text { colaboración }\end{array}$} & $60 \%$ & $40 \%$ & $y=0.749 x+0.875$ & Acepta: 0.749 \\
\hline & & $70 \%$ & $30 \%$ & $y=0.649 x+1.049$ & Acepta: 0.649 \\
\hline & & $80 \%$ & $20 \%$ & $y=0.542 x+1.105$ & Acepta: 0.542 \\
\hline \multirow{3}{*}{4} & \multirow{3}{*}{$\begin{array}{l}\text { H4: Interacción en } \\
\text { Schoology } \\
\text { colaboracion }\end{array}$} & $60 \%$ & $40 \%$ & $y=0.903 x+0.605$ & Acepta: 0.903 \\
\hline & & $70 \%$ & $30 \%$ & $y=0.847 x+0.694$ & Acepta: 0.847 \\
\hline & & $80 \%$ & $20 \%$ & $y=0.679 x+0.824$ & Acepta: 0.679 \\
\hline \multirow{3}{*}{5} & \multirow{3}{*}{$\begin{array}{l}\text { H5: Facilidad de } \\
\text { uso en Schoology } \\
\rightarrow \text { rol activo }\end{array}$} & $60 \%$ & $40 \%$ & $y=0.711 x+0.817$ & Acepta: 0.711 \\
\hline & & $70 \%$ & $30 \%$ & $y=0.739 x+0.729$ & Acepta: 0.739 \\
\hline & & $80 \%$ & $20 \%$ & $y=0.521 x+1.052$ & Acepta: 0.521 \\
\hline
\end{tabular}

Revista de Comunicación de la SEECI. 2021, nº 54, 19-41 
Salas-Rueda, R. A., Jiménez-Bandala, C-A. y Alvarado-Zamorano, C.

Schoology: plataforma web capaz de mejorar el proceso de enseñanza-aprendizaje en el nivel educativo superior

\begin{tabular}{|c|l|l|l|l|l|}
\hline \multirow{3}{*}{6} & H6: Interacción en & $60 \%$ & $40 \%$ & $y=0.634 x+0.951$ & Acepta: 0.634 \\
\cline { 2 - 6 } & Schoology $\rightarrow$ rol & $70 \%$ & $30 \%$ & $y=0.607 x+0.965$ & Acepta: 0.607 \\
\cline { 2 - 6 } & activo & $80 \%$ & $20 \%$ & $y=0.602 x+0.876$ & Acepta: 0.602 \\
\hline
\end{tabular}

Fuente: Elaboración propia por medio de la herramienta Rapidminer.

\subsection{Comunicación durante la creación del artículo científico}

Schoology facilita bastante $(n=12,44.44 \%)$, mucho $(n=10,37.04 \%)$, poco $(n=4$, $14.81 \%)$ y muy poco $(n=1,3.70 \%)$ la comunicación de los estudiantes durante la creación del artículo científico (Ver Tabla 1).

Los resultados del aprendizaje automático con $60 \%(0.326), 70 \%(0.302)$ y $80 \%$ (0.257) de entrenamiento indican que la hipótesis 1 es aceptada (Ver Tabla 2). Por lo tanto, la facilidad de uso en Schoology influye positivamente la comunicación de los estudiantes durante la creación del artículo científico.

La Figura 3 muestra el modelo predictivo 1 sobre el impacto de Schoology en el contexto educativo. Por ejemplo, si el estudiante piensa que la facilidad de uso en Schoology influye bastante durante el aprendizaje y es hombre entonces Schoology facilita bastante la comunicación de los estudiantes durante la creación del artículo científico.

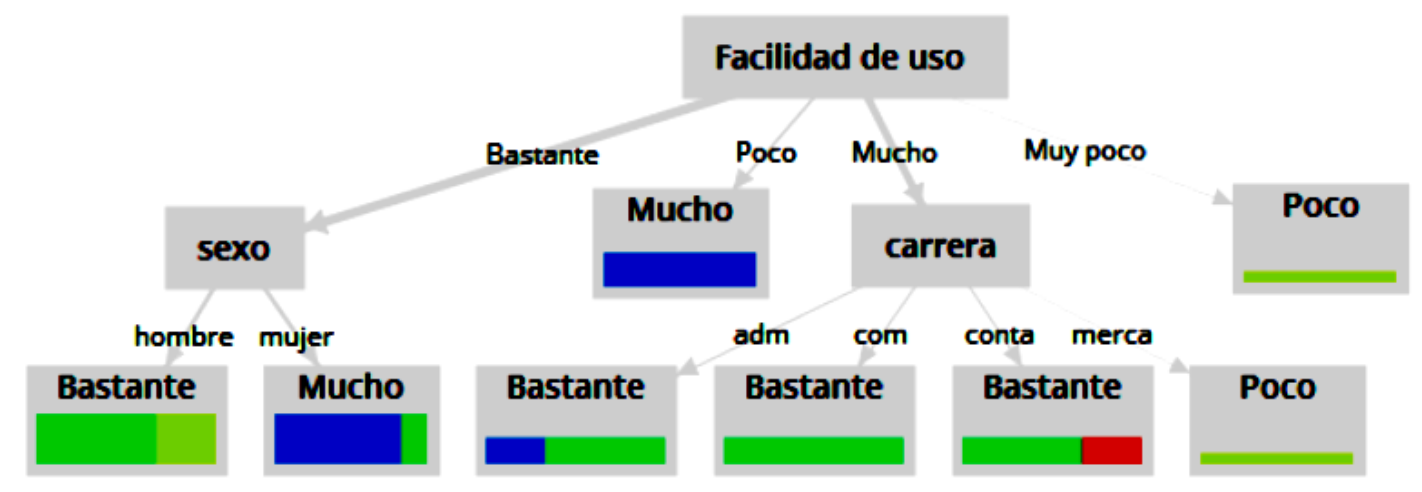

Figura 3. Modelo predictivo 1 sobre el uso de Schoology.

Fuente: Elaboración propia por medio de la herramienta Rapidminer.

El modelo predictivo 1 tiene una exactitud del $81.48 \%$ y presenta 8 condiciones sobre la facilidad de uso en Schoology y la comunicación (Ver Tabla 3). Por ejemplo, si el estudiante piensa que la facilidad de uso en Schoology influye mucho durante el aprendizaje y cursa la carrera de Administración entonces Schoology facilita bastante la comunicación de los estudiantes durante la creación del artículo científico. 
Salas-Rueda, R. A., Jiménez-Bandala, C-A. y Alvarado-Zamorano, C.

Schoology: plataforma web capaz de mejorar el proceso de enseñanza-aprendizaje en el nivel educativo superior

Tabla 3. Condiciones del modelo predictivo 1.

\begin{tabular}{|c|c|c|c|c|c|}
\hline No. & $\begin{array}{c}\text { Facilidad de uso } \\
\text { en Schoology }\end{array}$ & Sexo & Edad & Carrera & Comunicación \\
\hline 1 & Bastante & Hombre & - & - & Bastante \\
\hline 2 & Bastante & Mujer & - & - & Mucho \\
\hline 3 & Mucho & - & - & Administración & Bastante \\
\hline 4 & Mucho & - & - & Comercio & Bastante \\
\hline 5 & Mucho & - & - & Contaduría & Bastante \\
\hline 6 & Mucho & - & - & Mercadotecnia & Poco \\
\hline 7 & Poco & - & - & - & Mucho \\
\hline 8 & Muy poco & - & - & - & Poco \\
\hline
\end{tabular}

Fuente: Elaboración propia por medio de la herramienta Rapidminer

Por otro lado, los resultados del aprendizaje automático con $60 \%(0.326), 70 \%$ (0.368) y $80 \%(0.336)$ de entrenamiento indican que la hipótesis 2 es aceptada (Ver Tabla 2). Por lo tanto, la interacción en Schoology influye positivamente la comunicación de los estudiantes durante la creación del artículo científico.

La Figura 4 muestra el modelo predictivo 2 sobre el impacto de Schoology en el contexto educativo. Por ejemplo, si el estudiante piensa que la interacción en Schoology influye bastante durante el aprendizaje y estudia la carrera de Comercio entonces Schoology facilita bastante la comunicación de los estudiantes durante la creación del artículo científico.

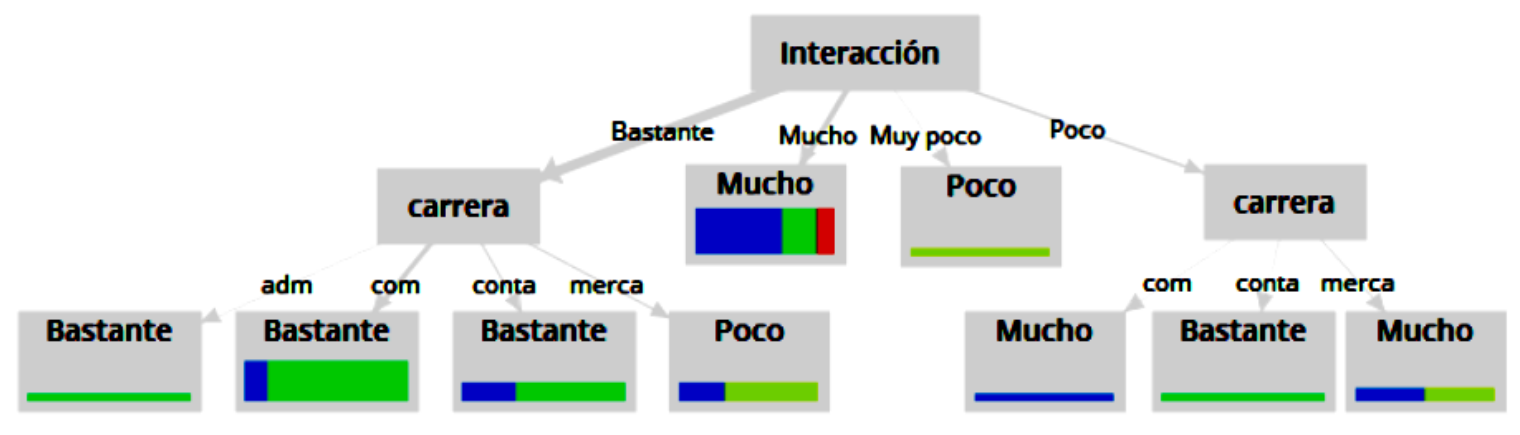

Figura 4. Modelo predictivo 2 sobre el uso de Schoology

Fuente: Elaboración propia por medio de la herramienta Rapidminer.

El modelo predictivo 2 tiene una exactitud del $74.07 \%$ y presenta 9 condiciones sobre la interacción en Schoology y la comunicación (Ver Tabla 4). Por ejemplo, si el estudiante piensa que la interacción en Schoology influye bastante durante el aprendizaje y estudia la carrera de Contaduría entonces Schoology facilita bastante la comunicación de los estudiantes durante la creación del artículo científico. 
Salas-Rueda, R. A., Jiménez-Bandala, C-A. y Alvarado-Zamorano, C.

Schoology: plataforma web capaz de mejorar el proceso de enseñanza-aprendizaje en el nivel educativo superior

Tabla 4. Condiciones del modelo predictivo 2

\begin{tabular}{|c|c|c|c|c|c|}
\hline No. & $\begin{array}{c}\text { Interacción en } \\
\text { Schoology }\end{array}$ & Sexo & Edad & Carrera & Comunicación \\
\hline 1 & Bastante & - & - & Administración & Bastante \\
\hline 2 & Bastante & - & - & Comercio & Bastante \\
\hline 3 & Bastante & - & - & Contaduría & Bastante \\
\hline 4 & Bastante & - & - & Mercadotecnia & Poco \\
\hline 5 & Mucho & - & - & - & Mucho \\
\hline 6 & Muy poco & - & - & - & Poco \\
\hline 7 & Poco & - & - & Comercio & Mucho \\
\hline 8 & Poco & - & - & Contaduría & Bastante \\
\hline 9 & Poco & - & - & Mercadotecnia & Mucho \\
\hline
\end{tabular}

Fuente: Elaboración propia por medio de la herramienta Rapidminer.

\subsection{Colaboración durante la creación del artículo científico}

Schoology facilita bastante $(n=11,40.74 \%)$, mucho $(n=10,37.04 \%)$, poco $(n=3$, $11.11 \%)$ y muy poco $(n=3,11.11 \%)$ la colaboración de los estudiantes durante la creación del artículo científico (Ver Tabla 1 ). Los resultados del aprendizaje automático con $60 \%(0.749), 70 \%(0.649)$ y $80 \%(0.542)$ de entrenamiento indican que la hipótesis 3 es aceptada (Ver Tabla 2). Por lo tanto, la facilidad de uso en Schoology influye positivamente la colaboración de los estudiantes durante la creación del artículo científico.

La Figura 5 muestra el modelo predictivo 3 sobre el impacto de Schoology en el contexto educativo. Por ejemplo, si el estudiante piensa que la facilidad de uso en Schoology influye bastante durante el aprendizaje y estudia la carrera de Mercadotecnia entonces Schoology facilita mucho la colaboración de los estudiantes durante la creación del artículo científico.

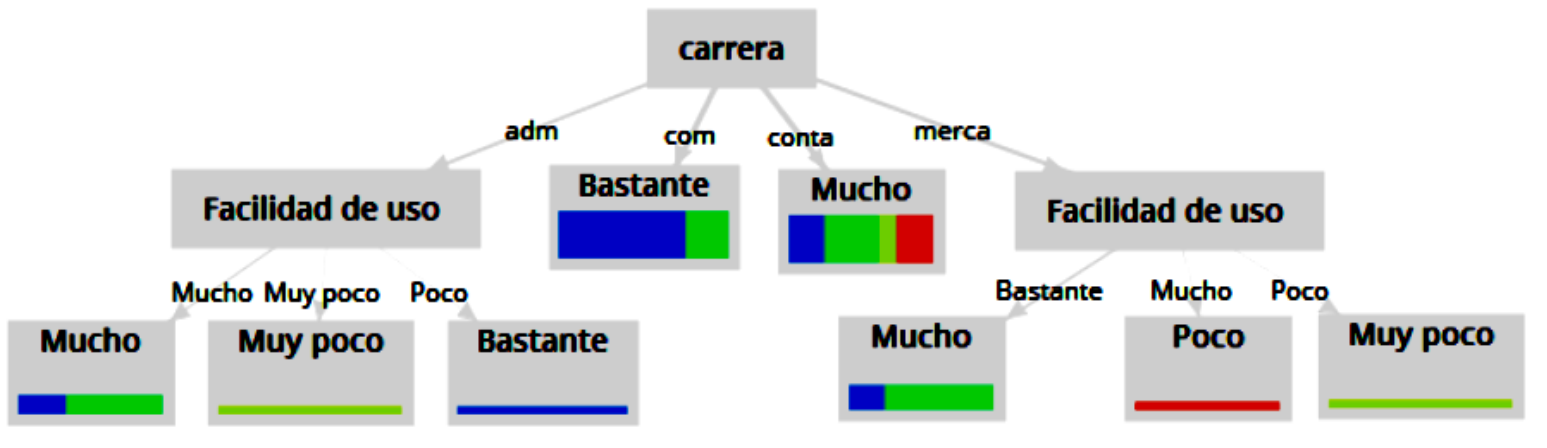

Figura 5. Modelo predictivo 3 sobre el uso de Schoology.

Fuente: Elaboración propia por medio de la herramienta Rapidminer.

El modelo predictivo 3 tiene una exactitud del $66.67 \%$ y presenta 8 condiciones sobre la facilidad de uso en Schoology y la colaboración de los estudiantes (Ver Tabla 5). Por ejemplo, si el estudiante piensa que la facilidad de uso en Schoology influye mucho durante el aprendizaje y estudia la carrera de Administración entonces 
Salas-Rueda, R. A., Jiménez-Bandala, C-A. y Alvarado-Zamorano, C.

Schoology: plataforma web capaz de mejorar el proceso de enseñanza-aprendizaje en el nivel educativo superior

Schoology facilita mucho la colaboración de los estudiantes durante la creación del artículo científico.

Tabla 5. Condiciones del modelo predictivo 3.

\begin{tabular}{|c|c|c|c|c|c|}
\hline No. & $\begin{array}{c}\text { Facilidad de uso } \\
\text { en Schoology }\end{array}$ & Sexo & Edad & Carrera & Colaboración \\
\hline 1 & Mucho & - & - & Administración & Mucho \\
\hline 2 & Muy poco & - & - & Administración & Muy poco \\
\hline 3 & Poco & - & - & Administración & Bastante \\
\hline 4 & - & - & - & Comercio & Bastante \\
\hline 5 & - & - & - & Contaduría & Mucho \\
\hline 6 & Bastante & - & - & Mercadotecnia & Mucho \\
\hline 7 & Mucho & - & - & Mercadotecnia & Poco \\
\hline 8 & Poco & - & - & Mercadotecnia & Muy poco \\
\hline
\end{tabular}

Fuente: Elaboración propia por medio de la herramienta Rapidminer.

Por otro lado, los resultados del aprendizaje automático con $60 \%(0.903), 70 \%$ (0.847) y $80 \%(0.679)$ de entrenamiento indican que la hipótesis 4 es aceptada (Ver Tabla 2). Por lo tanto, la interacción en Schoology influye positivamente la colaboración de los estudiantes durante la creación del artículo científico.

La Figura 6 muestra el modelo predictivo 4 sobre el impacto de Schoology en el contexto educativo. Por ejemplo, si el estudiante piensa que la interacción en Schoology influye bastante durante el aprendizaje y estudia la carrera de Comercio entonces Schoology facilita bastante la colaboración de los estudiantes durante la creación del artículo científico.

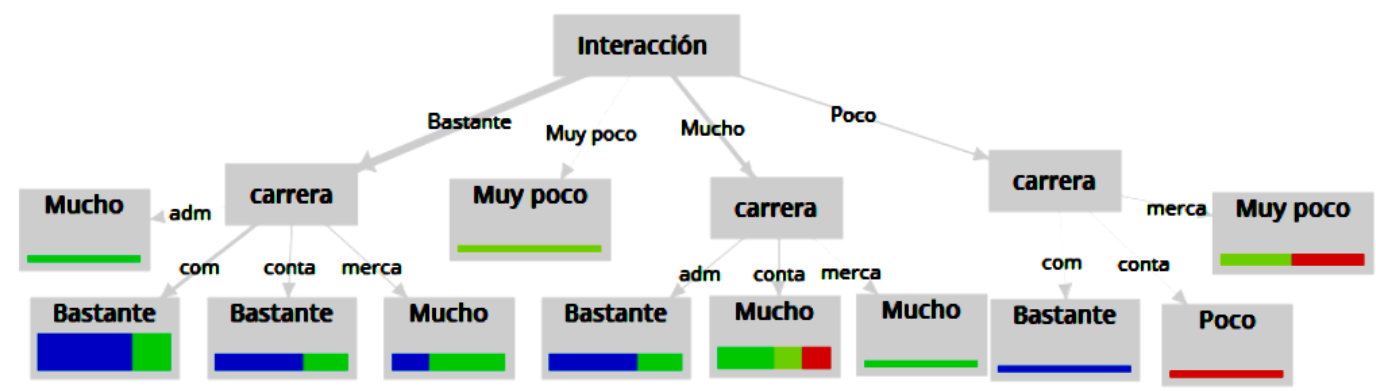

Figura 6. Modelo predictivo 4 sobre el uso de Schoology.

Fuente: Elaboración propia por medio de la herramienta Rapidminer.

El modelo predictivo 4 tiene una exactitud del $70.37 \%$ y presenta 11 condiciones sobre la interacción en Schoology y la colaboración de los estudiantes (Ver Tabla 6). Por ejemplo, si el estudiante piensa que la interacción en Schoology influye mucho durante el aprendizaje y estudia la carrera de Administración entonces Schoology facilita bastante la colaboración de los estudiantes durante la creación del artículo científico. 
Salas-Rueda, R. A., Jiménez-Bandala, C-A. y Alvarado-Zamorano, C.

Schoology: plataforma web capaz de mejorar el proceso de enseñanza-aprendizaje en el nivel educativo superior

Tabla 6. Condiciones del modelo predictivo 4.

\begin{tabular}{|c|c|c|c|c|c|}
\hline No. & $\begin{array}{c}\text { Interacción en } \\
\text { Schoology }\end{array}$ & Sexo & Edad & Carrera & Colaboración \\
\hline 1 & Bastante & - & - & Administración & Mucho \\
\hline 2 & Bastante & - & - & Comercio & Bastante \\
\hline 3 & Bastante & - & - & Contaduría & Bastante \\
\hline 4 & Bastante & - & - & Mercadotecnia & Mucho \\
\hline 5 & Mucho & - & - & Administración & Bastante \\
\hline 6 & Mucho & - & - & Contaduría & Mucho \\
\hline 7 & Mucho & - & - & Mercadotecnia & Mucho \\
\hline 8 & Poco & - & - & Comercio & Bastante \\
\hline 9 & Poco & - & - & Contaduría & Poco \\
\hline 10 & Poco & - & - & Mercadotecnia & Muy poco \\
\hline 11 & Muy poco & - & - & - & Muy poco \\
\hline
\end{tabular}

Fuente: Elaboración propia por medio de la herramienta Rapidminer.

\subsection{Rol activo durante la creación del artículo científico}

Schoology facilita bastante $(n=12,44.44 \%)$, mucho $(n=8,29.63 \%)$, poco $(n=5$, $18.52 \%)$ y muy poco $(n=2,7.41 \%)$ el rol activo de los estudiantes durante la creación del artículo científico (Ver Tabla 1). Asimismo, los resultados del aprendizaje automático con $60 \%(0.711), 70 \%(0.739)$ y $80 \%(0.521)$ de entrenamiento indican que la hipótesis 5 es aceptada (Ver Tabla 2). Por lo tanto, la facilidad de uso en Schoology influye positivamente el rol activo de los estudiantes durante la creación del artículo científico.

La Figura 7 muestra el modelo predictivo 5 sobre el impacto de Schoology en el contexto educativo. Por ejemplo, si el estudiante piensa que la facilidad de uso en Schoology influye mucho durante el aprendizaje y estudia la carrera de Mercadotecnia entonces Schoology facilita mucho el rol activo de los estudiantes durante la creación del artículo científico.

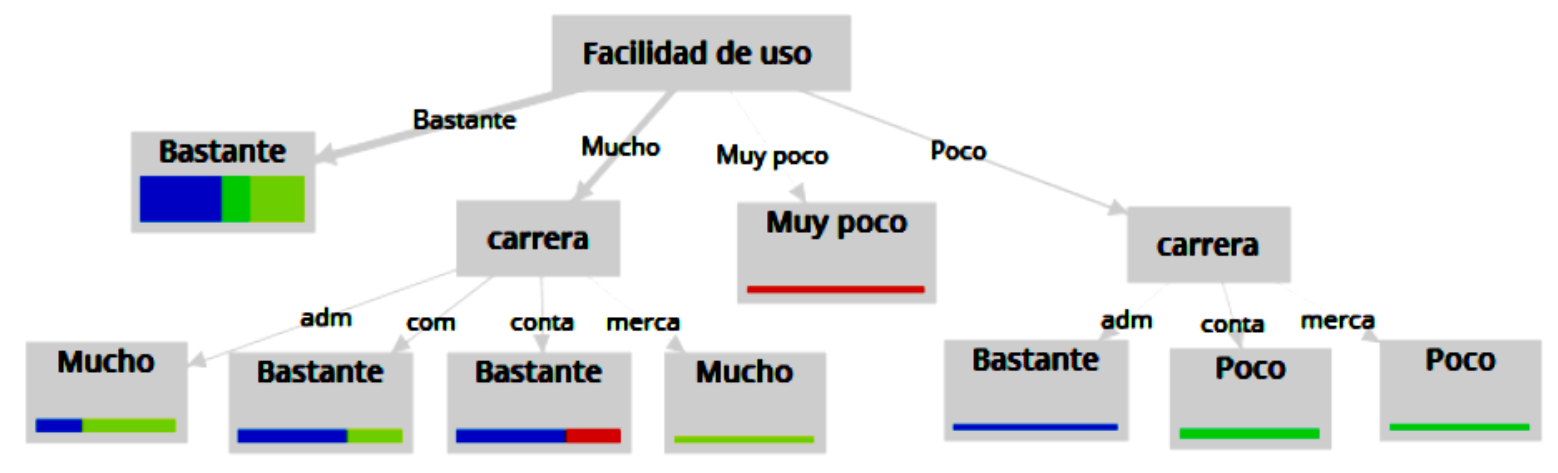

Figura 7. Modelo predictivo 5 sobre el uso de Schoology.

Fuente: Elaboración propia por medio de la herramienta Rapidminer.

El modelo predictivo 5 tiene una exactitud del $66.67 \%$ y presenta 9 condiciones sobre la facilidad de uso en Schoology y el rol activo de los estudiantes (Ver Tabla 
Salas-Rueda, R. A., Jiménez-Bandala, C-A. y Alvarado-Zamorano, C.

Schoology: plataforma web capaz de mejorar el proceso de enseñanza-aprendizaje en el nivel educativo superior

7). Por ejemplo, si el estudiante piensa que la facilidad de uso en Schoology influye mucho durante el aprendizaje y estudia la carrera de Administración entonces Schoology facilita mucho el rol activo de los estudiantes durante la creación del artículo científico.

Tabla 7. Condiciones del modelo predictivo 5.

\begin{tabular}{|c|c|c|c|c|c|}
\hline No. & $\begin{array}{c}\text { Facilidad de uso } \\
\text { en Schoology }\end{array}$ & Sexo & Edad & Carrera & Rol activo \\
\hline 1 & Bastante & - & - & - & Bastante \\
\hline 2 & Mucho & - & - & Administración & Mucho \\
\hline 3 & Mucho & - & - & Comercio & Bastante \\
\hline 4 & Mucho & - & - & Contaduría & Bastante \\
\hline 5 & Mucho & - & - & Mercadotecnia & Mucho \\
\hline 6 & Poco & - & - & Administración & Bastante \\
\hline 7 & Poco & - & - & Contaduría & Poco \\
\hline 8 & Poco & - & - & Mercadotecnia & Poco \\
\hline 9 & Muy poco & - & - & - & Muy poco \\
\hline
\end{tabular}

Fuente: Elaboración propia por medio de la herramienta Rapidminer.

Los resultados del aprendizaje automático con $60 \%(0.634), 70 \%(0.607)$ y $80 \%$ (0.602) de entrenamiento indican que la hipótesis 6 es aceptada (Ver Tabla 2). Por lo tanto, la interacción en Schoology influye positivamente el rol activo de los estudiantes durante la creación del artículo científico.

La Figura 8 muestra el modelo predictivo 6 sobre el impacto de Schoology en el contexto educativo. Por ejemplo, si el estudiante piensa que la interacción en Schoology influye bastante durante el aprendizaje y estudia la carrera de Comercio entonces Schoology facilita bastante el rol activo de los estudiantes durante la creación del artículo científico.

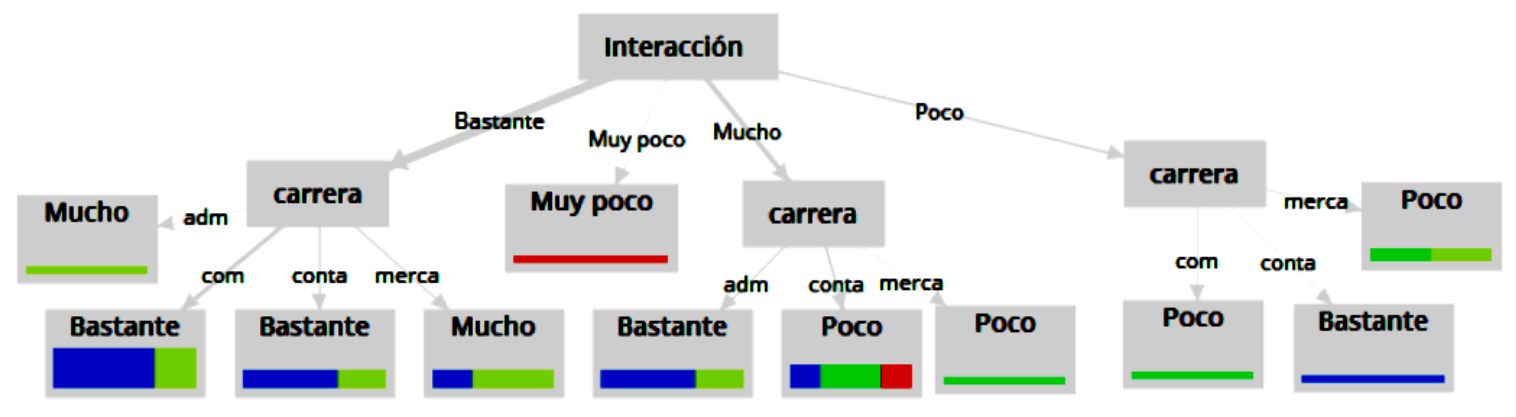

Figura 8. Modelo predictivo 6 sobre el uso de Schoology.

Fuente: Elaboración propia por medio de la herramienta Rapidminer.

El modelo predictivo 6 tiene una exactitud del $70.37 \%$ y presenta 11 condiciones sobre la facilidad de uso en Schoology y el rol activo de los estudiantes (Ver Tabla 8). Por ejemplo, si el estudiante piensa que la interacción en Schoology influye mucho durante el aprendizaje y estudia la carrera de Administración entonces 
Salas-Rueda, R. A., Jiménez-Bandala, C-A. y Alvarado-Zamorano, C.

Schoology: plataforma web capaz de mejorar el proceso de enseñanza-aprendizaje en el nivel educativo superior

Schoology facilita bastante el rol activo de los estudiantes durante la creación del artículo científico.

Tabla 8. Condiciones del modelo predictivo 6.

\begin{tabular}{|c|c|c|c|c|c|}
\hline No. & $\begin{array}{c}\text { Interacción en } \\
\text { Schoology }\end{array}$ & Sexo & Edad & Carrera & Rol activo \\
\hline 1 & Bastante & - & - & Administración & Mucho \\
\hline 2 & Bastante & - & - & Comercio & Bastante \\
\hline 3 & Bastante & - & - & Contaduría & Bastante \\
\hline 4 & Bastante & - & - & Mercadotecnia & Mucho \\
\hline 5 & Mucho & - & - & Administración & Bastante \\
\hline 6 & Mucho & - & - & Contaduría & Poco \\
\hline 7 & Mucho & - & - & Mercadotecnia & Poco \\
\hline 8 & Poco & - & - & Comercio & Poco \\
\hline 9 & Poco & - & - & Contaduría & Bastante \\
\hline 10 & Poco & - & - & Mercadotecnia & Poco \\
\hline 11 & Muy poco & - & - & - & Muy poco \\
\hline
\end{tabular}

Fuente: Elaboración propia por medio de la herramienta Rapidminer.

\subsection{Uso de Schoology en el proceso de enseñanza-aprendizaje}

Schoology mejora las condiciones de enseñanza-aprendizaje en la asignatura Taller de investigación debido a que esta plataforma web permite consultar y utilizar la información en cualquier momento y lugar:

"Schoology facilita bastante la creación y el entendimiento de las tareas" (Estudiante 13, mujer, 18 años, Contaduría).

"Si, porque es más fácil obtener los contenidos" (Estudiante 14, hombre, 18 años, Mercadotecnia).

"Si lo facilita, ya que ahí se sube todo" (Estudiante 16, mujer 18 años, Comercio).

"Facilitó la asimilación del conocimiento porque era más fácil recordar las actividades y había trabajos de apoyo" (Estudiante 18, mujer, 18 años, Comercio).

Los estudiantes de la asignatura Taller de investigación consideran que una de las ventajas de Schoology en el campo educativo es la facilidad y rapidez para realizar la entrega de tareas:

"Si, ya no sufrimos por imprimir las tareas" (Estudiante 1, hombre, 21 años, Mercadotecnia).

"Fue más rápido la entrega de tareas" (Estudiante 6, mujer, 17 años, Contaduría).

"Es más fácil entregar las tareas" (Estudiante 7, hombre, 19 años, Contaduría).

Otra de las ventajas relacionadas con el uso de Schoology en el campo educativo es la disponibilidad de la información: 
Salas-Rueda, R. A., Jiménez-Bandala, C-A. y Alvarado-Zamorano, C.

Schoology: plataforma web capaz de mejorar el proceso de enseñanza-aprendizaje en el nivel educativo superior

"Todo lo que vimos se quedó en la plataforma y eso fue de gran ayuda" (Estudiante 3, hombre, 19 años, Administración).

"Si, facilita asimilar el conocimiento porque la información está siempre a tu alcance para repasarla" (Estudiante 5, mujer, 18 años, Comercio).

"Podíamos buscar el contenido en cualquier momento" (Estudiante 6, mujer, 17 años, Contaduría).

Los estudiantes de las Licenciaturas en Administración, Comercio, Contaduría y Mercadotecnia consideran que los foros de discusión en Schoology facilitan el proceso de enseñanza-aprendizaje:

"Me parece que si. Con los foros de discusión" (Estudiante 1, hombre, 21 años, Mercadotecnia).

"Si, nos ayudó a entender los temas por medio de los foros de discusión" (Estudiante 14, hombre, 18 años, Mercadotecnia).

Asimismo, Schoology es una plataforma web educativa que facilita la realización de las actividades escolares:

"Estamos en una época donde la tecnología es fundamental" (Estudiante 9, hombre, 18 años, Comercio).

"Si, porque normalmente no se utilizan plataformas en otras materias" (Estudiante 13, mujer, 18 años, Contaduría).

"Si, es muy útil y práctica" (Estudiante 16, mujer 18 años, Comercio).

"Si, porque nadie utiliza esta plataforma" (Estudiante 17, mujer, 19 años, Contaduría).

Los alumnos de las Licenciaturas en Administración, Comercio, Contaduría y Mercadotecnia piensan que Schoology es una plataforma web útil para el proceso de enseñanza-aprendizaje:

"Si, porque así podemos ahorrar papel y darle un buen uso a la tecnología" (Estudiante 14, hombre, 18 años, Mercadotecnia).

"Si, porque podemos observar el material de apoyo" (Estudiante 16, mujer 18 años, Comercio).

"Sí, porque desde casa podíamos darnos una idea de lo que íbamos a ver en cada clase" (Estudiante 18, mujer, 18 años, Comercio).

La Figura 9 muestra la nube de palabra sobre el uso de Schoology en el proceso de enseñanza-aprendizaje. Las palabras con mayor frecuencia son enseñanza, fácil, educativo, aprendizaje, facilita, foros, tareas, información, plataforma, web y discusión. 


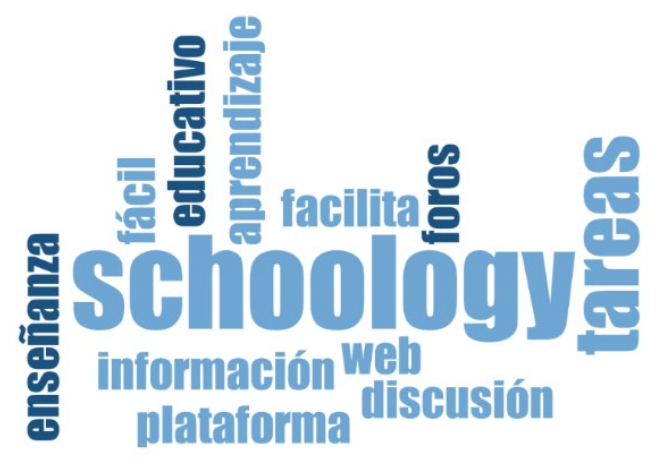

Figura 9. Nube de palabras sobre el uso de Schoology.

Fuente: elaboración propia.

\section{DISCUSIóN}

En el siglo XXI, los docentes están utilizando la tecnología para actualizar las actividades escolares dentro y fuera del salón de clases (Parsons et al., 2019; SalasRueda, Salas-Rueda y Salas-Rueda, 2019; Tang y Hew, 2019). Por ejemplo, Schoology es una plataforma web gratuita que las instituciones educativas pueden emplear para mejorar las condiciones de enseñanza-aprendizaje.

\subsection{Comunicación de los estudiantes}

Gran parte de los estudiantes ( $n=12,44.44 \%$ ) considera que Schoology facilita bastante la comunicación durante la creación del artículo científico. Asimismo, los resultados del aprendizaje automático sobre las hipótesis 1 y 2 superan el valor de 0.250, por lo tanto, la facilidad de uso e interacción en Schoology influyen positivamente la comunicación de los estudiantes durante la creación del artículo científico.

Los modelos predictivos 1 y 2 sobre el uso de Schoology y la comunicación de los estudiantes tienen una exactitud superior al $74.00 \%$. La técnica de árbol de decisión identifica 8 condiciones en el modelo predictivo 1 y 9 condiciones en el modelo predictivo 2. Por ejemplo, si el estudiante piensa que la facilidad de uso en Schoology influye bastante durante el aprendizaje y es hombre entonces Schoology facilita bastante la comunicación de los estudiantes durante la creación del artículo científico.

\subsection{Colaboración de los estudiantes}

La mayoría de los estudiantes ( $n=11,40.74 \%)$ piensan que Schoology facilita bastante la colaboración durante la creación del artículo científico. Asimismo, los resultados del aprendizaje automático sobre la hipótesis 3 y hipótesis 4 superan el valor de 0.540 , por lo tanto, la facilidad de uso e interacción en Schoology influyen positivamente la colaboración de los estudiantes durante la creación del artículo científico. 
Salas-Rueda, R. A., Jiménez-Bandala, C-A. y Alvarado-Zamorano, C.

Schoology: plataforma web capaz de mejorar el proceso de enseñanza-aprendizaje en el nivel educativo superior

Los modelos predictivos 3 y 4 sobre el uso de Schoology y la colaboración de los estudiantes tienen una exactitud superior al $66.60 \%$. La técnica de árbol de decisión identifica 8 condiciones en el modelo predictivo 3 y 11 condiciones en el modelo predictivo 4. Por ejemplo, si el estudiante piensa que la interacción en Schoology influye mucho durante el aprendizaje y estudia la carrera de Administración entonces Schoology facilita bastante la colaboración de los estudiantes durante la creación del artículo científico.

\subsection{Rol activo de los estudiantes}

La mayoría de los estudiantes ( $n=12,44.44 \%)$ consideran que Schoology facilita bastante el rol activo de los estudiantes durante la creación del artículo científico. Asimismo, los resultados del aprendizaje automático sobre las hipótesis 5 y 6 superan el valor de 0.520 , por lo tanto, la facilidad de uso e interacción en Schoology influyen positivamente el rol activo de los estudiantes durante la creación del artículo científico.

Los modelos predictivos 5 y 6 sobre el uso de Schoology y el rol activo de los estudiantes tienen una exactitud superior al $66.60 \%$. La técnica de árbol de decisión identifica 9 condiciones en el modelo predictivo 5 y 11 condiciones en el modelo predictivo 6. Por ejemplo, si el estudiante piensa que la interacción en Schoology influye mucho durante el aprendizaje y estudia la carrera de Administración entonces Schoology facilita bastante el rol activo de los estudiantes durante la creación del artículo científico.

Schoology permite modificar las funciones de los docentes y estudiantes durante el proceso de enseñanza-aprendizaje. Por ejemplo, los alumnos de las Licenciaturas en Administración, Comercio, Contaduría y Mercadotecnia utilizaron esta plataforma web para lograr la creación del artículo científico por medio de la consulta de la información, la discusión en los foros y la entrega de tareas.

Las plataformas web tienen un papel fundamental durante la planeación de los cursos y la realización de las actividades escolares dentro y fuera del salón de clases (Boloudakis, Retalis y Psaromiligkos, 2018; Elmaadaway, 2017; Yalcin y Kutlu, 2019). En particular, las instituciones educativas junto con los profesores tienen la oportunidad de utilizar Schoology durante el proceso de enseñanza-aprendizaje debido a que esta plataforma web educativa es gratuita y fácil de utilizar.

\section{CONCLUSIÓN}

Los avances tecnológicos como las plataformas web educativas están transformando la planeación, organización y realización de los cursos en el Siglo XXI. En particular, Schoology permite mejorar el proceso de enseñanza-aprendizaje por medio de la creación de nuevos espacios virtuales. Las ventajas sobre el empleo de Schoology en el campo educativo son la facilidad de uso, sencillez de su interfaz y disponibilidad de la información en cualquier momento y lugar. 
Salas-Rueda, R. A., Jiménez-Bandala, C-A. y Alvarado-Zamorano, C.

Schoology: plataforma web capaz de mejorar el proceso de enseñanza-aprendizaje en el nivel educativo superior

Los resultados del aprendizaje automático (regresión lineal) indican que la facilidad de uso e interacción en Schoology influyen positivamente la comunicación, la colaboración y el rol activo de los estudiantes durante la creación del artículo científico. De hecho, esta plataforma web educativa permite la consulta y el uso de los contenidos escolares en cualquier momento y lugar.

Asimismo, los estudiantes de las Licenciaturas en Administración, Comercio, Contaduría y Mercadotecnia consideran que Schoology facilita el proceso sobre la entrega de tareas y mejora la participación por medio de los foros de discusión. La ciencia de datos establece 6 modelos predictivos sobre el uso de esta plataforma en el campo educativo con una exactitud superior al $66.50 \%$. De hecho, la técnica de árbol de decisión identifica las relaciones entre el perfil del estudiante (sexo, edad y carrera) y el empleo de Schoology durante el proceso educativo (comunicación, colaboración y rol activo de los estudiantes).

Las limitaciones de esta investigación están relacionadas con el tamaño de la muestra y los temas de la asignatura Taller de Investigación. Por lo tanto, las futuras investigaciones tienen la oportunidad de analizar el impacto de Schoology en diversas áreas como Matemáticas, Medicina, Ingeniería y Salud.

Esta investigación mixta recomienda la incorporación de Schoology en las actividades escolares con la finalidad de fomentar el rol activo del estudiante durante el proceso de enseñanza-aprendizaje. Asimismo, esta plataforma web facilita la comunicación y colaboración de los estudiantes.

Las implicaciones de esta investigación impulsan a los docentes y las instituciones educativas a utilizar la tecnología como las plataformas web en las actividades escolares con la finalidad de lograr el aprendizaje centrado en el estudiante. En conclusión, Schoology es una plataforma web educativa que facilita el acceso de la información, la comunicación entre los participantes y la colaboración de los estudiantes durante el proceso de enseñanza-aprendizaje.

\section{REFERENCIAS}

Aldalalah, O., Ababneh, Z., Bawaneh, A. y Alzubi, W. (2019). Effect of Augmented Reality and Simulation on the Achievement of Mathematics and Visual Thinking Among Students. International Journal of Emerging Technologies in Learning, 14(18), 164-185. https://doi.org/10.3991/ijet.v14i18.10748

Bhagat, K., Wu, L. y Chang, C. (2019). The impact of personality on students' perceptions towards online learning. Australasian Journal of Educational Technology, 35(4), 98-108. https://doi.org/10.14742/ajet.4162

Boloudakis, M., Retalis, S. y Psaromiligkos, Y. (2018). Training Novice teachers to design moodle-based units of learning using a CADMOS-enabled learning design sprint. British Journal of Educational Technology, 49(6), 1059-1076. https://doi.org/10.1111/bjet.12678 
Salas-Rueda, R. A., Jiménez-Bandala, C-A. y Alvarado-Zamorano, C.

Schoology: plataforma web capaz de mejorar el proceso de enseñanza-aprendizaje en el nivel educativo superior

Byrne, J. (2019). Anytime Autonomous English MALL App Engagement. International Journal of Emerging Technologies in Learning, 14(18), 145-162. https://doi.org/10.3991/ijet.v14i18.10763

Cao, S. y Liu, H. (2019). Effectiveness Analysis of Edmodo-Based Blended English Learning Mode. International Journal of Emerging Technologies in Learning, 14(18), 64-75. https://doi.org/10.3991/ijet.v14i18.11184

Cerezo, R., Sánchez-Santillán, M., Paule-Ruiz, M. y Núñez, J. (2016). Students' LMS interaction patterns and their relationship with achievement: A case study in higher education. Computers \& Education, 96, 42-54. https://doi.org/10.1016/j.compedu.2016.02.006

Chung, C. y Ackerman, D. (2015). Student Reactions to Classroom Management Technology: Learning Styles and Attitudes Toward Moodle. Journal of Education for Business, 90(4), 217-223. https://doi.org/10.1080/08832323.2015.1019818

Costello, E. (2013). Opening up to open source: looking at how Moodle was adopted in higher education. Open Learning: The Journal of Open, Distance and eLearning, 28(3), 187-200. https://doi.org/10.1080/02680513.2013.856289

Deng, L. y Tavares, N. (2013). From Moodle to Facebook: Exploring students' motivation and experiences in online communities. Computers \& Education, 68, 167-176. https://doi.org/10.1016/j.compedu.2013.04.028

Dewi, F., Lengkanawati, N. y Purnawarman, P. (2019).Teachers' Consideration in Technology-Integrated Lesson Design - A case of Indonesian EFL Teachers. International Journal of Emerging Technologies in Learning, 14(18), 92-107. https://doi.org/10.3991/ijet.v14i18.9806

Dias, S., Hadjileontiadou, S., Diniz, J. y Hadjileontiadis, L. (2017). Computer-based concept mapping combined with learning management system use: An explorative study under the self- and collaborative-mode. Computers \& Education, 107, 127146. https://doi.org/10.1016/j.compedu.2017.01.009

Hendra-Divayana, D. (2019). The Implementation of Blended Learning with Kelase Platform in the Learning of Assessment and Evaluation Course. International Journal of Emerging Technologies in Learning, 14(17), 114-132. https://doi.org/10.3991/ijet.v14i17.8308

Li, G., Ouyang, S. y Yang, Y. (2019). A Study on the Construction of a Culture Pedagogical Network Learning Space - The CASH Curriculum Idea. International Journal of Emerging Technologies in Learning, 14(17), 73-85. https://doi.org/10.3991/ijet.v14i17.11235 
Salas-Rueda, R. A., Jiménez-Bandala, C-A. y Alvarado-Zamorano, C.

Schoology: plataforma web capaz de mejorar el proceso de enseñanza-aprendizaje en el nivel educativo superior

Parsons, D., Inkila, M. y Lynch, J. (2019). Navigating learning worlds: Using digital tools to learn in physical and virtual spaces. Australasian Journal of Educational Technology, 35(4), 144-159. https://doi.org/10.14742/ajet.3675

Salas-Rueda, R. (2018). Perspectivas de los estudiantes sobre la inclusión de videojuegos en el aprendizaje. International Journal of Educational Research and Innovation (IJERI), 10, 163-178. Recuperado de https://www.upo.es/revistas/index.php/IJERI/article/view/2613

Salas-Rueda, R. (2019). Construction and evaluation of a web application for the educational process on Normal Distribution considering the science of data and machine learning. Research in Learning Technology, 27, 1-24. https://doi.org/10.25304/rlt.v27.2085

Salas-Rueda, R., Salas-Rueda, E. y Salas-Rueda, R. (2019). Diseño y uso de una aplicación web para el campo de la estadística considerando el modelo assure y la ciencia de datos. Texto Livre: Linguagem e Tecnologia, 12(1), 1-24. https://doi.org/10.17851/1983-3652.12.1.48-71

Tang, Y. y Hew, K. (2019). Examining the utility and usability of mobile instant messaging in a graduate-level course: A usefulness theoretical perspective. Australasian Journal of Educational Technology, 35(4), 128-143. https://doi.org/10.14742/ajet.4571

Tazouti, Y., Boulaknadel, S. y Fakhri, Y. (2019). ImALeG: A Serious Game for Amazigh Language Learning. International Journal of Emerging Technologies in Learning, 14(18), 28-37. https://doi.org/10.3991/ijet.v14i18.10854

Yalcin, M. y Kutlu, B. (2019). Examination of students' acceptance of and intention to use learning management systems using extended TAM. British Journal of Educational Technology, 50(5), 2414-2432. https://doi.org/10.1111/bjet.12798

Yundayani, A., Kardijan, D. y Herawan, T. (2019). Integrating ICT in English for Academic Purposes Materials through Task-Based Approach. International Journal of Emerging Technologies in Learning, 14(17), 29-42. https://doi.org/10.3991/ijet.v14i17.10753

Zhang, M. y Li, Y. (2019). Students' Continuance Intention to Experience Virtual and Remote Labs in Engineering and Scientific Education. International Journal of Emerging Technologies in Learning, 14(17), 4-15. https://doi.org/10.3991/ijet.v14i17.10799

Zou, J., Liu, Q. y Yang, Z. (2012). Development of a Moodle course for schoolchildren's table tennis learning based on Competence Motivation Theory: Its effectiveness in comparison to traditional training method. Computers \& Education, 59(2), 294-303. https://doi.org/10.1016/j.compedu.2012.01.008 
Salas-Rueda, R. A., Jiménez-Bandala, C-A. y Alvarado-Zamorano, C.

Schoology: plataforma web capaz de mejorar el proceso de enseñanza-aprendizaje en el nivel educativo superior

\section{AUTORES:}

\section{Ricardo-Adán Salas-Rueda}

Doctor en Diseño de Nuevas Tecnologías, Maestro en Administración e Ingeniero en Sistemas Electrónicos. Investigador de Tiempo Completo en el Instituto de Ciencias Aplicadas y Tecnología, Universidad Nacional Autónoma de México. Investigador Nacional SNI nivel 1 (CONACYT).

ricardo.salas@icat.unam.mx

Orcid ID: http://orcid.org/0000-0002-4188-4610

\section{Carlos-Alberto Jiménez-Bandala}

Doctor en Economía. Profesor-Investigador de la Facultad de Negocios en la Universidad La Salle México, Investigador nacional SNI nivel 1.

carlos jimenez@lasalle.mx

Orcid ID: https://orcid.org/0000-0003-4431-0054

\section{Clara Alvarado-Zamorano}

Doctora en Investigación en Didáctica de las Ciencias Experimentales y las Matemáticas. Técnico Académico Titular $\mathrm{C}$ en el Instituto de Ciencias Aplicadas y Tecnología, Universidad Nacional Autónoma de México. PRIDE D. SNI C.

clara.alvarado@icat.unam.mx 